\title{
Analysis of Influencing Factors in Metacognition in English Writing
}

\author{
Yingnan Liu \\ Changchun University of Science and Technology, Changchun, China
}

\begin{abstract}
Metacognition in writing is the cognitive monitoring system in the process of writing. The essence of the system is self-management and self-control of self-consciousness. It can be embodied in the process of the writer acquiring information and analyzing the information to monitor the cognitive process correctly and immediately and choosing the suitable strategy to monitor the writing, to persist in or change the method or manner. So this paper is try to make a discussion on matecognition in writing and its influencing factors, analyze the information and sum up the general features of metacognition in writing in purpose of furnishing English writing.
\end{abstract}

Index Terms - metacognition, English writing, motivation, self-determination

\section{INTRODUCTION}

English writing is a process which involves various elements and is a process which is monitored by metacognition. Writing as one of students' important skill and a way of communication, plays a significant role in English teaching and learning. A requirement of English writing ability would become one of the important criteria for qualified personal in the new century. Therefore, it is quite urgent for English teachers to help students improve their writing competence. But the present situation of writing teaching in Chinese universities is far from satisfactory. On one hand, most English teachers still focus teaching on correcting mistakes and instructing students to imitate and write according to some models rather than help the students to develop their own creativeness when the students start to learn writing. Although the students practice a lot and the teachers correct many times, the level of students' writing seem to stay at the same. On the other hand, most of the students' writing lack of original creation especially when they are in junior and senior. So their writing is nearly identical in though pattern and textual structure. Therefore, improving students writing ability has become one of the key tasks for English teachers.

In the field of language studying, many linguists have emphasised the importance of learners' self-autonomy. Numerous empirical studies have been shown to be supportive of the importance of self-concept of ability, perceived value of the subjects, and expectations for future success as crucial predictors of academic performance.

To understand the role of motivation's influencing factor in English writing, the definition of motivation may be discussed in the first place. Motivation referred to learners' directed reinforcing effort in learning a language; which was the effort that a language learner willing to pay in the process of second language learning. Since the goal of learners may be different, some wish to become linguists, some for future job, and others wish to be identified with the foreign country. Therefore, the effort learners make may be different according to their individual goals in language learning. The former learner goals can be viewed as what Brown mentioned the instrumental orientation, which referred to acquiring language for instrumental goals, such as passing an entrance exam, communicating with foreigners. While the latter learner goals can be viewed as the integrative orientation, with which can be referred to learners' desire to be accepted or identified as members of the group of the target language, which means they were open to the new language and its people.

Different learner needs and interests may result in different degree of effort they are willing to make in the language-learning process. Individual differences in their willingness and perseverance to learn a language were closely linked to achievement needs. Also, the need for achievement was composed of three factors, including the person's desire for success (or failure), the value of the task as a motivation, and the method used toward success. In other words, it was proposed that motivation including both integrative and instrumental, was highly related to the individual needs for achievement or their goals toward learning the target language.

\section{Motivation}

Motivation, according to Dornyer (1998), was the "process whereby a certain amount of instigation force arises, initiates action, and persists"(p.122).as long as there is no other forces weakening it until the planned goals were reached. However, motivation can be defined as the degree to which an individual tries to learn the language because of their willingness to do so and satisfaction obtained from such activity, such definition focuses on learners' inner desire and attitude toward language learning. Furthermore, based on Deci and Ryan's self-determination theory, motivation was related to all aspects of "activation" and "intention", including energy, direction, persistence and equifinality. 
Motivation was classified as extrinsic motivation and intrinsic motivation based on the degree of self-determination. From extrinsic motivation to intrinsic motivation, they were classified as five categories: external regulation, introjected regulation, identified regulation, integrated regulation, and intrinsic motivation.

External regulation means that learners perform certain behaviors so as to satisfy external demands or to get external reward. These external regulated behaviors were usually experienced as being controlled or alienated.

Introjected regulation, which was another type of extrinsic motivation remained quite controlling. People who were introjected-regulated mean that they performed the behaviors with a feeling of pressure, avoiding guilt, or obtaining self-esteem. For example, children may do homework not because they like it; but because they avoid being punished by teachers, or get other students' approval.

Identified regulation, though still classified as extrinsic motivation, was a more autonomous form of extrinsic motivation. Identified regulation occurred when people identified the importance of some behaviors and took it as his or her own value. For example, a girl who learned English because she knew the importance of learning English, and she viewed this as her own goals in her life.

The most self-determined form of extrinsic motivation, integrated regulation, referred to that people has fu11y assimilated the identified regulation to themselves. In other words, integrated regulation occurred when people taking the new regulation in their life and make it congruent with their own needs and values. Though integrated regulation and intrinsic motivation were said to be similar in some way, integrated regulation was sti11 viewed as one of the types of extrinsic motivation, since people with integrated motivation were regarded as doing behaviors out of the presumed external value which was separable from the behaviors.

Intrinsic motivation occurred when someone was moved to do something for fun or challenge, instead of environmental factors. In other words, people decided to do the action or behavior because of their inherent interest toward the activity, rather than because of the external outcome.

\section{SELF-DETERMINATION THEORY}

Self-determination theory was an organismic theory focused on human behavior and its correlation to social context. Human beings were viewed as "proactive organism" whose intrinsic motivation may be either facilitated or undermined by the social context in self-determination theory. The focus of the theory was on the investigation of human beings' inherent tendencies as well as the psychological needs, which were said to be the basic elements for self-motivation and integration in personality. In self-determination theory, it was pointed out that there was close relationship between people's motivational behaviors and the social contextual factors. For example, when people feel relatedness, secure, or a sense of competence from the environment, then their motivation for doing the behavior may become autonomous instead of being controlled.

In self-determination theory, motivation can be classified as mainly two types: intrinsic and extrinsic. Intrinsic motivation referred to people doing something out of the inherent interest or joy; whereas extrinsic motivation referred to doing something because it contributed to a separable outcome. However, the two types of motivation were not opposite site of each other; instead, they were along a continuum. From motivation to external compliance, and to self commitment, they were classified as motivation, external regulation, introjected regulation, identified regulation, integrated regulation, and intrinsic motivation. External regulation, introjected regulation, identified regulation, and integrated regulation were different forms of extrinsic motivation. In other word, whether people do the action out of inherent interest on the task, or out of external control, ex: getting reward, avoid of punishment, or to attain ego esteem.

Dcei and Ryan proposed that human intrinsic motivations developed from three primary psychological needs: need for competence, need for autonomy (or self-determination), in which one have choice or voice for his/her own action, and need for relatedness, searching for connection with others as well as the social world. Empirical evidences supporting the idea that intrinsic and extrinsic motivation can be used in predicting learners' achievement in second language learning. It was claimed that the more internalized students are in writing, the more persistent students can be, implying that students' degree of internalization may determine their long-term learning outcomes.

\section{Mode of Motivation Theory}

Different theories have different views in regards to the component and construct of motivation. The theories can be classified intro three groups. The first group of theories includes Freud's psychoanalytic theory and Hull's drive theory, both of which hold the view that stress reduction has significant impact on behavior. The second group of theories were proposed by Weiner, which includes Lewin's field theory, Atkinson's achievement theory and Rotter's social learning theory. As per the three of the theories, behavior is highly dependent on the individual's desire for success and his/her incentives to achieve the intended result. Even though these are quite different theories, they both hold the view that human beings try to understand themselves and their surroundings and that growth processes are a necessary part of human motivation.

The psychoanalytic theory is from Freud's perception of individuals, who struggles between their instinctive needs and the restrictions imposed by society. The laws of motivation stems from the analysis of this conflict between their instinctive drives and their inhibitions. Although there are studies which do not support the Freudian view, the primary 
processes of though and functioning of the "self" have generated important hypotheses which are still valid today.

The drive theory tries to explain individual's behavior by a series of impulses which from habits. The subjects' behavior is explained by means of a sequence of impulses or acquired behaviors which are generated from the action of certain incentives or stimuli. Hull is one of the most well-known representatives. The primary components of this theory are: First comes anxiety. It is an emotional reaction which works by means of production rejection of the agent which generates stress. The learning procedure of those individuals who are affected by this type of behavioral forces is strengthened by tasks of difficulty and reduced by more complicated tasks. Second is conflict. The "component of avoidance" is more important than the "component of approximation", which stimulates motivational procedure. Third is frustration. This happens when the target is not reached. Fourth is the principle of social facilitation. This means the reaction of other individuals in the subject's conduct or performance.

Research on drives has achieved success in explaining the behaviors of animals with the most basic needs (for example, thirst or hunger), their reaction to stimuli, their mechanical learning process to satisfy these needs, however, in other areas, it hasn't achieved the same degree of success. It seems quite obvious that the greatest contribution of the theory is the accurate and systematic research of motivated conduct from a purely mechanical point of view.

The achievement theory is based on environmental factors such as the student's personal experiences or their desire to achieve good performance. Students who are motivated by achievement opt for problems which pose a certain amount of challenge, make an effort to find solutions to these difficult problems before giving up. They will lose motivation if they solve the problem easily and try to find more challenging or innovative tasks. These students usually get better marks than other students with similar IQs. On the other hand, students who are motivated by the desire to avoid failure tend to select easier problems. They will become discouraged by mistakes and motivated by success. They prefer friendly classmates and respond best to tasks which pose less of a challenge.

It seems that people are more intrinsically motivated when they are small and tend to engage themselves in achievement-related behaviors. However, as they grow up and reach higher educational levels, they tend to be more extrinsically motivated because of the increased significance of external rewards. It's clear that achievement theory has made valuable contributions, however, it has also obvious limitations. It disregards the underlying values of the individual, focusing only on need achievement and not taking into account his or her personal preferences as decisive factors in task selection. Schiefele states: "Researcher of achievement motivation disregard the content to be learned. Emphasis on student performance overlooks the possibility that students come to like their subjects and learn because they value the process of being emerged in certain areas of knowledge" (1991, p.19). The social learning theory upholds that the mental process of cognition affect our behavior. Its adherents maintain that individuals learn through imitation, that they can distinguish between motivating and non-motivating circumstances, that they set up expectations related to achievement and that they perceive the world in a subjective manner.

Attribution theories reflect the causes of an event, the information which affected its causal deduction, and the results of the causal attribution. Internal causes comprise IQ, physical appearance, personality, aptitude, effort and health. External causes include the difficulty of an activity, external help and luck. Ability is regarded as more constant than effort.

The distinction by Heider (1958) is that between internal and external factors which determine behavior:

\section{A. Strongly Motivated Individuals}

Attribute their success in activities of great capacity and effort.

Persist when facing failure, ascribing it to lack of effort, something which is changeable.

Select tasks with average difficult as they generate more self-assessable feedback.

Make great efforts, as they believe that it is what contributes to success.

\section{B. Individuals with Weak-low Motivation}

Engaged in few activities, as they attribute success to external factors instead of internal factors and do not regard effort as its cause.

Give up quickly when facing failure, as they consider the latter as being caused by lack of capacity, which is uncontrollable.

Select either easy or very difficult tasks, as they may not generate self-assessable feedbacks.

Make little effort as they do not believe it is what contributes to success.

The attribution theory is an obvious component of cognitive functionalism and it emphasizes the individual's causal cognition of their motivational states. Sometimes those causes are attributed to the subject himself or herself (IQ, effort, personality, etc.) and on other circumstances, they are attributed to environmental factors (the difficulty of tasks, school and parental influence, etc.). Weiner distinguishes different types of factors which have obvious influence on the subjects' behavior, causal antecedents, causal attributions, causal dimension, prospects of success, motivational conducts.

Several investigations have emphasized the fundamental role played by individual's opinions, beliefs and perceptions. Scholars of this field pointed out that to have a positive vision of oneself and of the learning situations is essential to attain expected performance. Learners may experience various successes and failures in a school environment which influence their beliefs and expectations, as well as their cognitive structures, thus change their frame of reference to 
interpret, understand and organize the information they possess of themselves and others. Therefore, studying the students' beliefs can provide us with insights into their conduct and academic performance.

We can conclude from the above studies that we should teach our students to attribute their success to controllable factors such as effort instead of to the uncontrollable factors. This will eventually break the vicious circle of failure. The idea of re-educating students has led teachers to change the type of input provided to students. The feedback should try to establish links between the efforts the students has made to the results. Comments such as "your work has improved because you have made an effort" or "I can see you have studied a lot for the exam" can help students attribute their success to controllable factors.

The humanistic theory stems from the humanistic psychology. Human beings are subjects instead of objects of study. Each person should be perceived in terms of personal conscience, which compromises subjective experience and the way in which the individual sees and evaluates him or herself. One of the most well-known humanistic psychologists states that everyone tries to reach a state of self-realisation. However, the latter can be facilitated or hindered by our interactions with others. The approval of others helps self-realisation and also our opinions about ourselves and our attitudes towards others are closely linked.

\section{OTHER INFLUENCING FACTORS OF METACOGNITION IN ENGLISH WRITING}

In addition to the above factors that we have discussed, there are also some other important factors which also play a significant role in English writing. Now, we will discuss them one by one.

\section{A. Learning Environment}

Autonomous language learning does not simply mean learns learn by themselves. Instead, they need external supports in the process of developing autonomy, for example, the changes of institutional mechanism which includes teachers, learning facilities, learning materials, etc. Since we are mainly concerned with developing an autonomous pedagogy in the classroom, in which teacher plays an important role, the discussion here will focus on teacher.

In accordance with the concept of learner autonomy, autonomous learners need to be able to decide on their learning needs and goals, identify learning resources, choose and carry out proper learning strategies, organize the learning tasks and self-evaluate their learning results, all of which will be very hard or even impossible to be accomplished by the learners in the highly competitive learning environment infested with exams the "didactic learning modes" with the teacher as its center. In China, however, though the reform of college English pedagogy has been in practice for years, teachers seem to be still swimming comfortably in the sea of traditional English teaching, being the center of the classroom, emphasizing knowledge of English over competence to use English. The assessment system designs and implements many school-based and public examinations which can only make students to learn by rote.

\section{B. English Proficiency of Learners}

English Proficiency of learners is a direct factor to influence the metacognition in writing. For example, students with medium level will have a gap in comparing with the high level students in writing strategies. In answering the question "What will do when you don't know how to carry out your writing?" Students with high level will say that they could unscramble their thoughts and find out the solutions. They may leave for a few minute and come back soon. But they will not give up. But students with a medium level will ask a high level student or teacher for help or give up immediately. Some scholars who make research on the writing and teaching find out that the responses of the high level students, medium level students and low level students will be different in writing. From the example, we know that level of English writing is not isolated from other aspects levels of English learners but closely related with them. For example, The level of reading may not only affect whether the students are able to understand the requirement of a writing task or not but also has a great impact on their understanding when they read some sample writing materials. Other aspects of English levels such as listening can also have an important influence on English writing. So in order to improve the writing level of students, teachers should not focus on the writing level itself but also make effort to improve the overall levels of students as a whole. Only by this, the writing levels of students can be improved.

\section{Metacognition in Native Language Writing}

Because Chinese is an old language which belongs to Sino-Tibetan language family, it has significant difference with English which belongs to Indo-European language family. In learning processes, listening and reading don not disturb evidently because they are input activity which receive the information passively while speaking and writing are disturbed terribly by native language as output. In writing process, the influence of the native language can not be neglect. We should decrease the negative influence and increase the positive ones to help our writing.

\section{The Score Criteria in Writing Tests}

Generally speaking, the criterion of "effectively addresses the writing task, demonstrates a well developed logical organizational structure with clearly stated main ideas and sufficient supporting details, has almost no errors of vocabulary, spelling, punctuation or syntax" is understood as correct forms of language. Students' compositions may become the media of reflection of the students' language levels but not a communicative tool. Some scholars argued that if teachers applied this criterion to the writing lessons, there must appear the following two questions: 
The whole writing teaching process will become a process of correcting students' composition, which is only to find out the mistakes and giving scores. But in the point of view of the modern pedagogy, mistake is the inevitable outcomes of the learning processes. The compositions that students hand in are the beginning of writing process. And only by correcting the content, structure and the languages repeatedly, the students' writing ability can be improved. The writing exercise is not equals to the writing test. Using the criteria of scoring the writing test to require the writing exercise and only give a grade but do not give students time to considerate and evaluate violate the rule writing processes. Writing is not a one-off activity. It needs to be retrospect. To emphasize too much on the grammatical mistakes will make students neglect the contents of composition.

Teachers give more emphasis on the surface mistakes when they explain writing, but do not concern the arrangement of the concrete, the skill of making sentences and the rhetoric skills. When students write a composition they may also not consider the communicative aims but only consider the language forms. In this case, the value of writing will be missed.

\section{CONCLUSION}

To make a whole look at factors of metacognition in English writing, we can see that both writing activity and teaching activity must have instruction of metacognition. They effect interdependently in the learning processes of writing.Language learners should learn to recognize their learning strategies, and teachers should advise them to select the most appropriate methods for instructional environment. Successful learners can inform students who are not so successful in language learning in regards to learning strategies, techniques and skills. Through monitoring and coaching each other, students can take an active role in not only learning but also teaching.

\section{REFERENCES}

[1] Brown, G. (1995). Listening to Spoken English, (2nd edn). London: Longman.

[2] Deci, E. L. \& Ryan, R. M. (1985). Intrinsic motivation and self-determinaton in human behaviour. New York: Plenum.

[3] Dornyer Z. (1998). Motivation in Second and Foreign Language Learning. Language Learning, 3,117-137.

[4] Flavell, J. H. (1979). Metacognition and cognitive monitoring: A new area of cognitive-developmental inquiry. American Psychologist, 34, 906 - 911.

[5] Heider, F. (1958). The psychology of interpersonal relations. New York: Wiley.

[6] Hull, C. (1933). Hypnosis and Suggestability: An Experimental Approach. New York: Appleton-Century-Crofts.

[7] Ryan, R. M., \& Deci, E. L. (2000). Self-determination theory and the facilitation of intrinsic motivation, social development, and well-being. American Psychologist, 22, 29-31.

[8] Schiefele,U. (1991). Interest, learning, and motivation. Educational Psychologist, 3, 17-22.

Yingnan Liu was born in Jiutai, Jilin Province, China on April 14 th, 1980, who has got a degree of Master of Arts in foreign linguistics and applied linguistics from Changchun University of Science and Technology, Changchun, Jilin Province, China in 2006.

As a lecture, he teaches English intensive reading and listening at School of Foreign Languages, Changchun University of Science and Technology. Previous research interests are English Methodology. At present he focuses on Curriculum and ESL teaching. 